\title{
Love wave dispersion in anisotropic visco-elastic medium
}

\author{
S. M. Gir SUbhash* - V.K. GaUR ${ }^{\star *}$
}

Received on March 7th, 1978

\section{ABSTRACT}

The paper presents a study on Love wave propagation in a anisotropic visco-elastic layer overlying a rigid half space. The characteristic frequency equation is obtained and the variation of the wave number with frequency under the combined effect of visco-elasticity and anisotropy is analysed in detail. The results show that the effect of visco-elasticity on the wave is similar to that of anisotropy as long as the coefficient of anisotropy is less than unity.

\section{RIASSUNTO}

La nota presenta uno studio sulla propagazione delle onde di Love in uno strano anisotropo visco-elastico che giace su uno spazio semi rigido. $E^{\prime}$ stata ricavata l'equazione caratteristica della frequenza, ed è stata

* L'Istitute de physique du Globe - 5, rue Rene Descartes, U.L.P. Strasbourg 67084 (France).

** Dept. of Geology and Geophy - U.O.R. ROORKEE (U.P.) - India. 
analizzata dettagiamente la variazione del numero d' onda con la frequenza, sotto l'effetto combinato della visco-elasticità e dell'anisotropia. I risultati mostrano che l'effetto della visco-elasticità sul numero d'onda è simile a quello dell'anisotropia, finchè il coefficiente di anisotropia è inferiore all'unità.

\section{INTRODUCTION}

Study of the propagation behaviour of surface waves in a visco-elastic and anisotropic models is of great interest for the accurate inversion of the observed surface wave data. The results of numerical model studies of Schwab and Knopoff (1972), for a anelastic medium, and those of Cramping and Taylor (1971), for anisotropic medium, shown that both these factors can have a considerable influence on the propagation behaviour of surface waves. In order to understand their propagation behaviour in more realistic conditions it will be interesting to study their propagation in visco-elastic anisotropic medium.

In the present paper we examine the dispersion behaviour of Love wave in a simple two layer model, consisting of a visco-elastic (Voigt type) anisotropic layer overlying a rigid half space. The results are then compared with those of Negi and Upadhyay (1968) who have studied the effect of anisotropy on the Love wave dispersion, for a similar model.

\section{Mathematical Formulation}

The geometry of the problem is illustrated in Fig. 1. Rectangular co-ordinate system is considered with the $Z$-axis directed vertically downward and the $X-Y$ plane coinciding with the free surface. Considering Love wave propagation in the positive $X$-direction, neglecting body forces and assuming small defor- 
mations, the equation of motion of Love wave in a visco-elastic anisotropic layer can be written as:

$$
\delta \frac{\bar{o}^{2} v}{\partial t^{2}}=\left(N+N^{\prime} \frac{\delta}{\partial t}\right) \frac{\delta^{2} v}{\partial x^{2}}+\left(L+L^{\prime} \frac{\delta}{\partial t}\right) \frac{\delta^{2} v}{\delta x^{2}}
$$

where,

$N$ is the modulus of rigidity and $N^{\prime}$ is the coefficient of visco-elasticity in the $X$-direction.

and

$L$ and $L^{\prime}$ are the corresponding quantities in the $Z$-direction.

In representing a harmonic wave travelling in the $X$-direction we choose a solution of the following form:

$$
\vartheta=V(Z) \exp [i(\omega t-K X)]
$$

where, $V(Z)$ is areal function of $Z$ and $K$ is a complex quantity equal to $(k+i k)$, whose real part represents the wave number and the imaginary part represents the damping coefficient. Putting this value of $V(Z)$ in equation [1] we get:

$$
\left(L+i L^{\prime}\right) \frac{\mathrm{d}^{2} Y}{\mathrm{~d} Z}+\left[\rho \omega^{2}-K^{2}\left(N+i N^{\prime} \omega\right)\right] V=0
$$

The above equation reduces to:

$$
\frac{\mathrm{d}^{2} V}{\mathrm{~d} Z}+S^{2} V=0
$$

and its solution can be written as:

$$
V=A \exp (\imath S Z)+B \exp (-i S Z)
$$

where, 
$A$ and $B$ are constants and $S$ is complex quantity given by the relation:

$$
S^{2}=\frac{\rho \omega)^{2}-K^{2}\left(N+i N^{\prime} \omega\right)}{L+i L^{\prime}(\omega)}
$$

Boundry conditions of zero stress at the free surface and of zero displacement ot the interface require that:

$$
\frac{\mathrm{d} V}{\mathrm{~d} Z} 0=\quad \text { at } \quad Z=0
$$

and

$$
V=0 \quad \text { at } Z=H
$$

From equations $[5,7]$ and $[8]$ we get:

$$
A=B
$$

and

$$
A \exp (i S H)+B \exp (-i S H)=0
$$

equations [9] and [10] yield,

or

$$
\begin{aligned}
& \exp (2 i S H)=-1 \\
& S^{2} H^{2}=(n+1 / 2)^{2} \lambda^{2}
\end{aligned}
$$

putting the value of $S^{2}$ in equation [10] and separating into real and imaginary parts, we get:

$$
\begin{gathered}
R H^{2}\left[w_{n}^{2}\left(1 / \beta_{o}^{2}-a Q\right)-a-b w_{n} Q(p-1)\right] / C+ \\
-i R H^{2}\left[w_{n}^{2}\left(\frac{Q w_{n}}{\beta^{2}}+b Q^{2}\right)+b+a w_{n} Q(p-1)=\right. \\
=C\left(n+\frac{1}{2}\right)^{2} \lambda^{2}
\end{gathered}
$$


where,

$$
\frac{L}{L}=Q \quad \text { and } \quad \frac{N}{N^{\prime}}=p Q
$$

By definition (Yanakawa and Sato, 1964), 1/Q represents the specific dissipation factor for shear wave.

$$
R=N / L \quad, \quad \sqrt{N / \rho} \quad, \quad C^{\prime}=\left(1+Q^{2} w_{n}{ }^{2}\right)
$$

and

$$
a=k_{\mathrm{o}}^{2}-k_{\mathrm{1}}^{2} \quad, \quad b=2 k_{\mathrm{o}} k_{1}
$$

equating real and imaginary parts and putting $g=1$, we get:

$$
\begin{gathered}
R H^{2}\left[\left(w_{n} / \beta_{\mathrm{o}}\right)^{2}-a C^{\prime}\right]=(n+1 / 2) \lambda^{2} C^{\prime} \\
\frac{w_{n}^{3} Q}{\hat{\beta}_{\mathrm{o}}{ }^{2}}+b C^{\prime}=0
\end{gathered}
$$

equations [14] and [15] yield:

$$
\begin{gathered}
a=\frac{w_{n}^{2}}{C^{\prime} \beta_{\mathrm{o}}^{2}}-\frac{(n+1 / 2)^{2} \lambda^{2}}{R H^{2}} \\
b=\frac{w_{n}^{3} Q}{C^{\prime} \beta_{o}{ }^{2}}-
\end{gathered}
$$

wave number $k_{\mathrm{o}}$ an damping coefficient $k_{1}$ for various modes are given by the relations:

$$
\begin{aligned}
& k_{\mathrm{o}}(n)=\sqrt{\frac{1}{2}\left(\sqrt{a^{2}+b^{2}}+a\right)} \\
& k_{l}(n)=\sqrt{\frac{1}{2}\left(\sqrt{a^{2}+b^{2}}-a\right)}
\end{aligned}
$$


Equation [18], considered along with [16] and [17] shows $k_{o}(n)$ woul be real for all values of $w$ and thus the propagation should take place at all frequencies without any cut off. However, when $Q \ll 1$ (as is the case for real earth), the contribution of ' $b$ ' at low frequencies will be negligible and therefore the dispersion behaviour of Love waves will be same as for elastic earth.

3. WAVE NUMBER VARIATION AND THE EFFECT OF VISCO-ELASTICITY ON THE PHASE GROUP VELOCITIES

Equations [18] along with [16] and [17], has been used to analyse the wave number and, phase and group velocities for following two cases, assuming $Q=50$. The value of $Q$ of this order for upper crust have been reported by Mitchell (1973).

\subsection{Isotropic visco-elastic case $(R=1)$}

From the results given in Table 1, it can be readily observed that for a visco-elastic surface layer, the value of $k_{\mathrm{o}} H$ is smaller than its corresponding value for a perfectly elastic layer. The effect of visco-elasticity, is therefore to decrease the wave

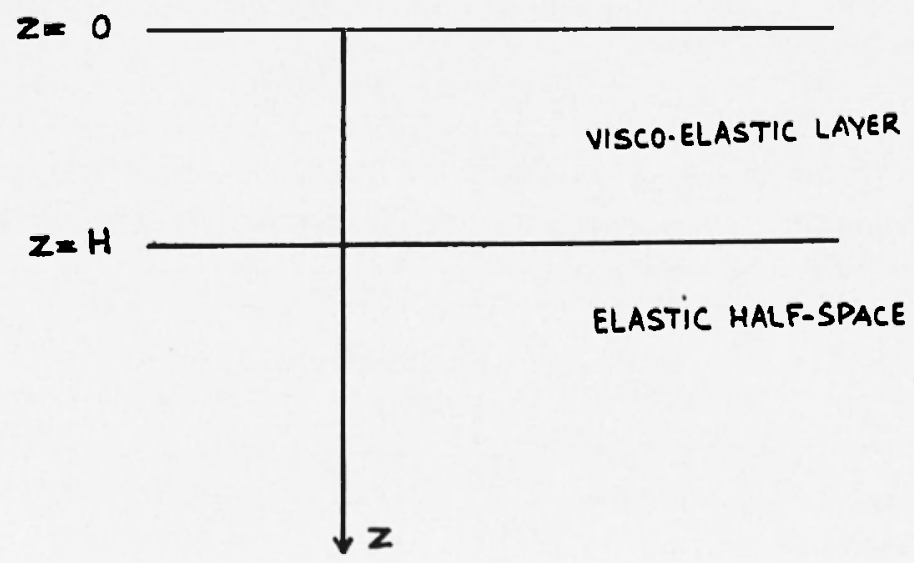


number. This result is qualitatively similar to that brought about by anisotropy $(R<1)$ ), (Negi and Upadhyay, 1968 except that in the case of visco-elasticity, the effect goes on increasing with frequency.

To study the effect of visco-elasticity on the phase and group velocities, equation [14] has been analysed and the results are shown in Fig. 2. Unlike the perfect elastic case in which the phase and group velocities converge to a common limit as $w_{n} \rightarrow^{\circ} \cdot C$ (Hudson, 1962); in the present case the phase and group velocities start diverging after a certain value of $w_{n}$, because of the increasing effect of visco-elasticity. However, at low frequencies when the effect of visco-elasticity is small, the phase and group velocities follow the same trend as found in the case of a perfectly elastic case.

\subsection{Anisotropic visco-elastic case}

a) For $R>1$ - Comparing the results given in column 5 and 6 of table 2, with those in column 2 of the same table, it can be observed that, whereas the effect of anisotropy is to increase the $k_{0}$ value, visco-elasticity tends to decrease it. Two effects therefore counteract each other. Since the effect of visco-elasticity goes on increasing with frequency, the two effects will annull each other at a frequency $w_{c}$ (say).. For frequencies higher than $w_{e}$, the visco-elastic effect will be predominent and there will be a net decrease in $k_{0} H$ under the combined effect of two. This is qualitatively equivalent to the effect of a anisotropic elastic layer with $R<1$, (Negi and Upadhyay, 1968). Thus under the combined effect of visco-elasticity and anisotropy with $R>1$, the medium appears as being simply anisotropic with coefficient of anisotropic $R<1$.

b) For $R<1-$ Since the effect of both viesco -elasticity and anisotropy with coefficient $R<1$, is to decrease the $k_{o} H$ value the combined effect of two is to enhance the effect of each other. The results computed for $R=0.69$ are shown in column 3 and 4 of Table 2. 
S. M. GIR SUBHASH - V. K. GAUR

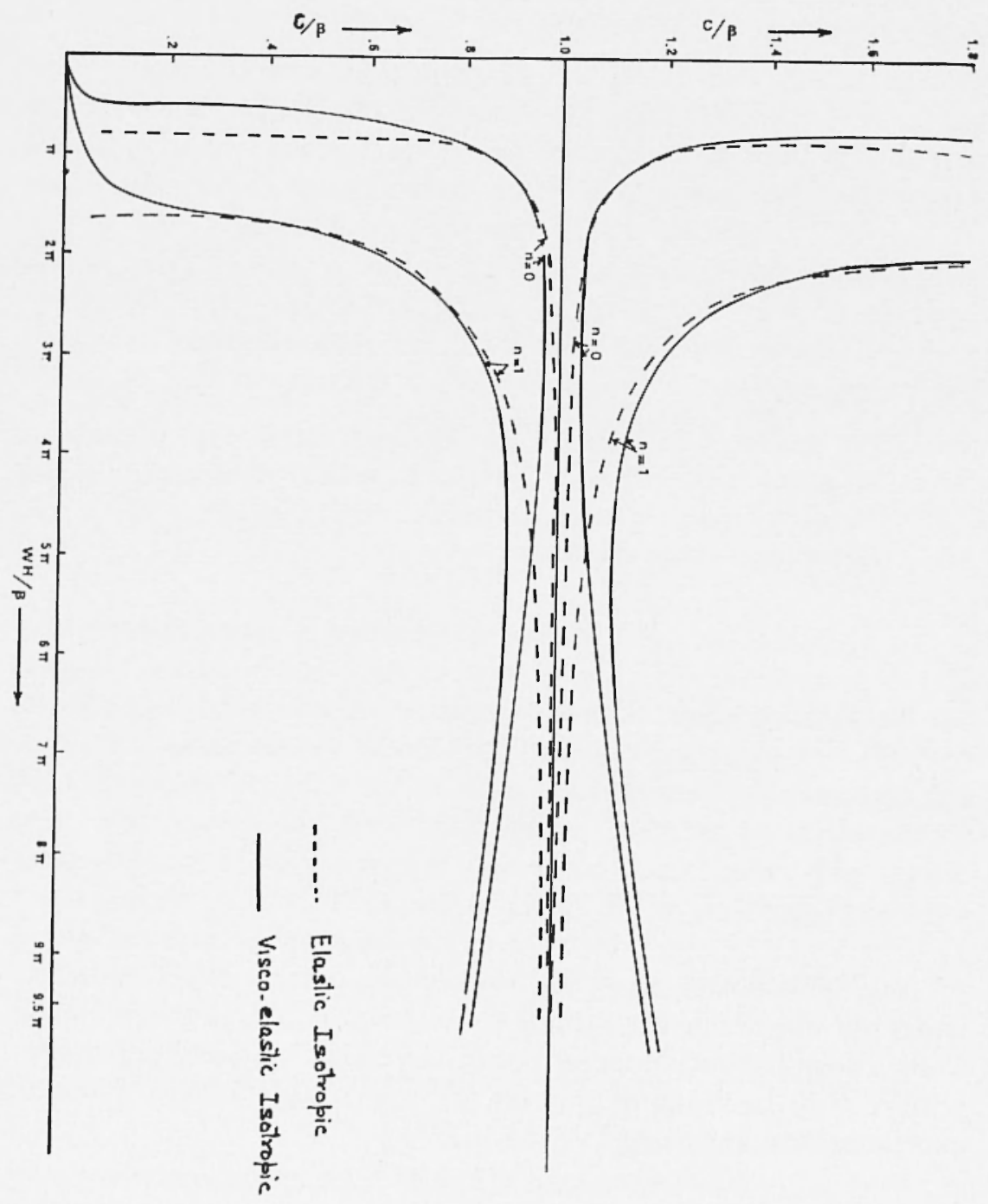




\section{EQUivalENCE OF PHASE VELOCITY}

At a frequency $w_{e}$ where the effect of visco-elasticity and anisotropy with coefficient $R>1$, annul each other, the waves will propagate with same phase velocity as they will do in a isotropic elastic medium.

Equating $k_{e}$ value for a wave propagating with frequency $w_{e}$ in a visco-elastic anisotropic medium, eq. [18], to its corresponding value in the isotropic elastic model (Hudson, 1962 eq. [23] we get:

$$
\frac{1}{2}\left(\sqrt{a^{2}+b^{2}}+a\right)=\left(\frac{w_{e} H}{\beta_{0}}\right)^{2}-\left(n+\frac{1}{2}\right)^{2} \lambda^{2}
$$

Subtituting the values of $a$ and $b$ in the above equation we get:

$$
\begin{gathered}
4 R^{2}\left[C^{2}(M-P)^{2}-(M-P) M C^{3}\right]+ \\
+4(M-P) C^{3} P R-M^{2} J^{2}=0
\end{gathered}
$$

where,

$$
M=\left(w_{e} H / \beta_{o}\right)^{2}, P=(n+1 / 2) \text { and } J=w_{e} Q
$$

therefore we get

$$
R=\frac{P \pm \sqrt{p^{2}+M^{2} J^{2}\left[\left(1 / C^{4}\right)-M /(M-P) C^{3}\right]}}{2\left[\frac{(M-P)-M}{C}\right]}
$$

From the above equation one can deduce the value of the coefficient of anisotropy which will annul the known visco-elastic effect at a given frequency. 


\section{CONCLUSION}

From the above discussion of the results it can be concluded that the effect of the visco-elasticity on the wave number variation with frequency is similar to that produced by anisotropy with coefficient $<1$. When the coefficient of anisotropy is $>1$, the effect of visco-elasticity is reflected as a decrease in the effect of anisotropy. For a given frequency there will be a combination of the coefficients of visco-elasticity and anistropy which will cancel the effect of each other and the propagation will take place as in isotropic elastic medium.

\section{ACKNOWLEDGEMENTS}

The authors are thankful to the Head of the department of Geology and Geophysics for providing funds for the computations of the results. 
LOVE WAVE DISPERSION IN ANISOTROPIC ECC.

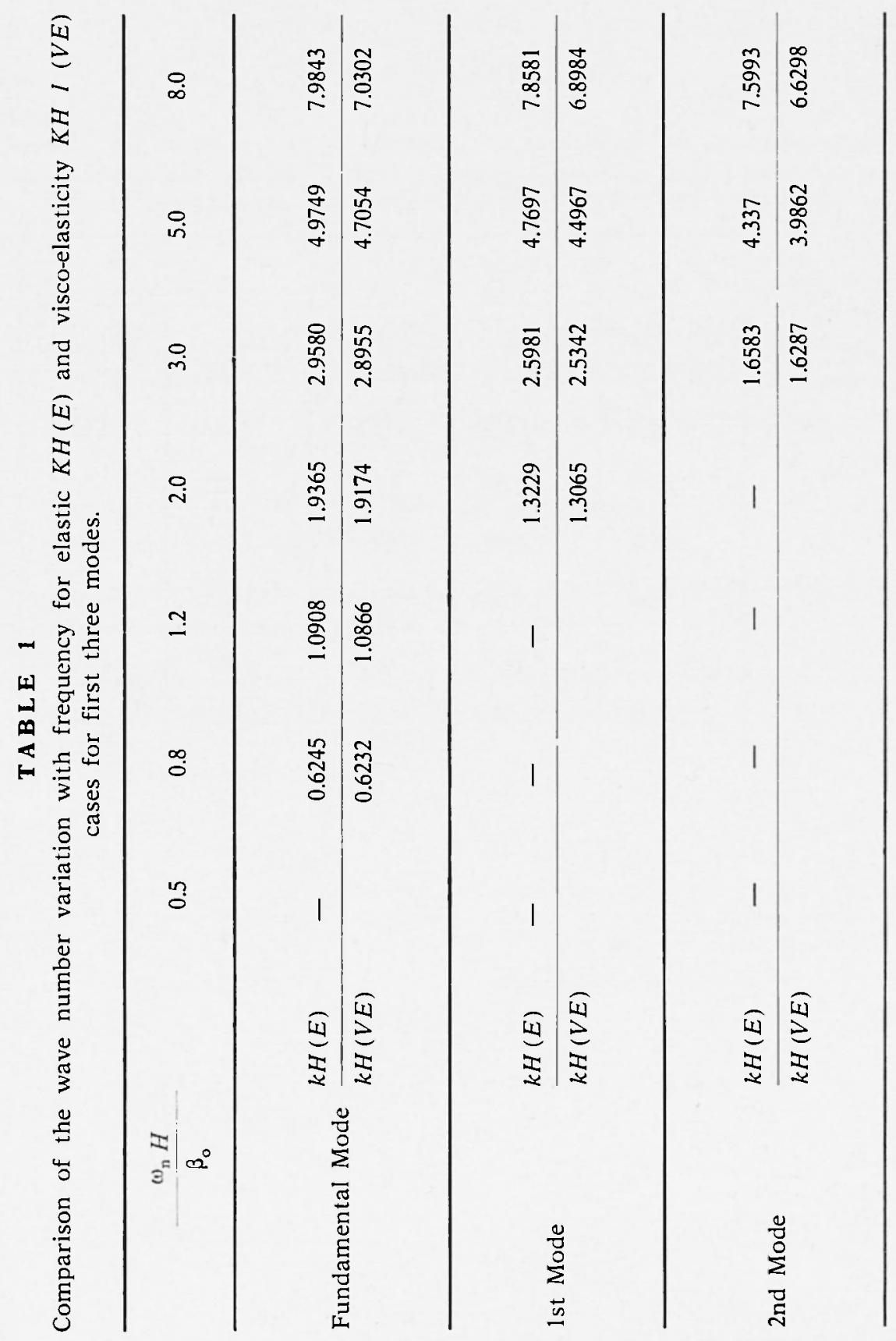




\section{TA B L E 2}

Comparison of the wave number variation with frequency for elastic isotropic $K H(E), R=1.0$, anisotropic $K H(E A), R=0.6844$ and visco-elastic anisotropic $K H(V E A), L / L^{\prime}=50$ cases for fundamental mode.

\begin{tabular}{|c|r|r|r|r|r|}
\hline \multirow{2}{*}{$\omega_{n} H$} & \multirow{2}{*}{$R=1.0$} & \multicolumn{2}{|c|}{$R=0.68$} & \multicolumn{2}{|c|}{$R=1.44$} \\
\cline { 2 - 5 }$\beta_{n}$ & $K H(E)$ & $K H(E A)$ & $K H(V E A)$ & $K H(E A)$ & $K H(V E A)$ \\
\cline { 3 - 6 } 0.8 & 0.6245 & 0.5219 & 0.5208 & 0.6829 & 0.6816 \\
1.2 & 1.0908 & 1.0355 & 1.0313 & 1.1253 & 1.1211 \\
1.6 & 1.5198 & 1.4806 & 1.4707 & 1.5447 & 1.5349 \\
2.0 & 1.9364 & 1.9058 & 1.8867 & 1.9561 & 1.9371 \\
2.5 & 2.4494 & 2.4253 & 2.3885 & 2.4650 & 2.4284 \\
3.0 & 2.9580 & 2.9380 & 2.8754 & 2.9709 & 2.9085 \\
& & & & &
\end{tabular}




\section{REFERENCES}

CRAMPING S., TAYLOR D.B., 1971 - Propagation of surface waves in anisotropic media. "Geophy. J.R. Astr. Soc. », 24, pp. 71-87.

Hudson J.A., 1962 - Love wave in hetrogeneous media. "Geophy. J.R. Astr. Soc. ", 6 pp. 131-147.

NEGI J.G., UPADHYAY S.K., 1968 - Effect of anisotropy on Love wave propagation. B.S.S.A., pp. 257-266.

Mitchell B.J., 1973 - Surface wave attenuation and crustal anelasticity in central north America. B.S.S.A., 63. pp. 1057-1071.

Schwb F.A., KNoporf L., 1972 - Fast surface wave and free mode com. putation. Methods in computation Physics. "Academic Press ", N. Y. 